\title{
Relaciones Transversales Faciales en Niños Chilenos de la Región del Maule
}

\author{
Transverse Face Relations in Chilean Children from Maule Region
}

"Nicolás Alejandro Ramos Pazos; "Iván Claudio Suazo Galdames; **Magda Lucía Martinez Martinez \& *Lorena Reyes

RAMOS, P. N. A.; SUAZO, G. I. C.; MARTINEZ, M. M. L. \& REYES, L. Relaciones transversales faciales en niños chilenos de la Región del Maule. Int. J. Morphol., 25(4):703-707, 2007.

RESUMEN: Las relaciones transversales faciales presentan variaciones en las distintas etnias, su conocimiento resulta relevante en el campo de las cirugías plástica y maxilofacial, la antropología y la medicina forense. En el presente estudio se analizan las relaciones transversales faciales de 45 niños mesofaciales de la Región del Maule, Chile, mediante un análisis cefalométrico frontal y la confección de modelos del paladar. Fueron analizados las dimensiones: ancho transpalatino, ancho maxilar, ancho facial y ancho mandibular. Las dimensiones encontradas fueron superiores a las descritas en la literatura. Se encontró que el ancho transpalatino mostraba una mediana correlación con el ancho maxilar, no así con el ancho facial. No se observó dimorfismo sexual en la muestra.

PALABRAS CLAVE: Relaciones faciales; Cara, Antropología Física; Medicina Forense.

\section{INTRODUCCIÓN}

Las relaciones de las medidas antropométricas craneales entregan importantes antecedentes para la Antropología Física y Medicina Forense (del Sol, 2005); y son de gran utilidad en el campo de la Ortodoncia y la Cirugía Ortognática (Quevedo \& Jeldes, 2004).

Los análisis antropométricos cefálicos consideran mediciones en sujetos vivos u osamentas, realizadas de manera directa o mediante estudios en radiografías laterales o frontales (Ricketts, 1981; Ghafari et al., 1995; Vellini, 2002).

Para el estudio de las relaciones transversales que establecen los componentes esqueléticos y dentoalveolares de la cabeza, se utilizan principalmente los análisis frontales, siendo el de Ricketts el más ampliamente utilizado (Grummons \& Kappeyne, 1987; Gregoret, 1997).

Las relaciones entre las dimensiones transversales de los arcos dentales y las características faciales han sido analizadas por diversos autores. Para Chaconas (1982) los arcos dentales del paciente dolicofacial son angostos y pueden estar asociados a una bóveda palatina alta; por otro lado Moyers (1992) manifiesta que hay poca correlación entre anchos de arco y cualquier medición de ancho esquelético o facial.

Snodell et al. (1993) analizaron las relaciones verticales y transversales en 25 hombres y 25 mujeres de raza blanca de 4 a 25 años de edad, determinando que las proporciones entre las mediciones transversales del esqueleto facial a los 6 años de edad fueron similares a las medidas en la edad adulta, no ocurriendo lo mismo con las medidas verticales. Estos autores encontraron, además cierto dimorfismo sexual en el valor del ancho facial en niños de 9 años, lo que discrepa con los resultados de Huertas \& Ghafari (2001) quienes observaron que el ancho mandibular fue semejante en niños y niñas a los 10 años de edad.

Águila (1998) realizó un análisis del ancho transpalatino en adultos blancos y cubanos negroides, encontrando diferencias entre ambos grupos estudiados; con valores mayores para los negroides que para los caucásicos. Estos resultados concuerdan con los estudios de Björk (1951), Jacobson \& Rosestein (1970), Kowalski et al. (1974), Fonseca \& Klein (1978) y Burris \& Harris (2000), quienes comprobaron la existencia de diferencias significativas entre etnias.

\footnotetext{
* Unidad de Anatomía Normal, Facultad de Ciencias de la Salud, Universidad de Talca, Chile.

** Departamento de Pediatría Estomatológica, Facultad de Ciencias de la Salud, Universidad de Talca, Chile.
} 
Para Gravilovic (1967) la filiación étnica de la población chilena del Valle Central es compleja. Dentro de ella la presencia de población indígena Mapuche en la Región del Maule, alcanza un $95.08 \%$ del total de la población indígena (Gundermann et al., 2005); para García \& Lips (1986a, 1986b), la ascendencia étnica de individuos chilenos determina diferencias en el índice cefálico. Del sol determinó el índice cefálico en 50 individuos de grupo étnico mapuche a través del diámetro transversal biparietal y bitemporal, con un promedio de $148 \mathrm{~mm}$ y $141 \mathrm{~mm}$, respectivamente.

En el presente estudio se describen las relaciones transversales faciales y se analiza la presencia de dimorfismo sexual en estas relaciones, en un grupo de niños y niñas mesofaciales de la Región del Maule, Chile.

\section{SUJETOS Y MÉTODO}

En el estudio participaron 45 pacientes de la Clínica del Niño del Centro de Clínicas Odontológicas de la Universidad de Talca, Chile, 22 de sexo femenino y 23 de sexo masculino, con edades de entre 7 y 10 años (Promedio 8,3 años, D.S. 0,95), diagnosticados previamente como individuos mesofaciales de acuerdo al análisis frontal descrito por Ricketts.

En estos pacientes se realizaron modelos de yeso de la arcada maxilar luego de una impresión de alginato. El ancho transpalatino fue medido en la intersección del surco lingual con el margen gingival de los primeros molares superiores permanentes contralaterales. Se utilizó un orthometro de Korkhaus (lectura mínima $0,1 \mathrm{~cm}$ ), los datos fueron registrados en una tabla de registro de anchos transpalatinos utilizando 1 decimal.
Posteriormente en las radiografías cefalométricas frontales estrictas se realizó un trazado cefalométrico manual sobre una lámina de acetato, siguiendo los métodos y criterios establecidos por Ricketts para el análisis frontal (Ricketts; Gregoret), midiendo los anchos maxilar, mandibular y facial.

El ancho maxilar corresponde a una línea horizontal, que une puntos bilaterales ubicados en la intersección de los contornos de la tuberosidad del maxilar y pilar cigomático (Spillane \& McNamara, 1989; Quevedo \& Jeldes). El ancho mandibular corresponde a una línea horizontal, que une puntos ubicados en la parte más alta de la incisura antegonial (Spillane \& McNamara; Quevedo \& Jeldes). El ancho facial corresponde a una línea horizontal, que une puntos ubicados en el centro de la raíz del arco cigomático (Spillane \& McNamara; Quevedo \& Jeldes). Fig. 1.

La muestra fue analizada por 2 observadores, un ortodoncista y un investigador entrenado. Mediante el método de la doble medición, se establecieron los valores de medición de error interobservador, con un rango observado de 0,4 a $1,1 \mathrm{~mm}$.

Utilizando el programa Microsoft Excel 2000 se calcularon las estadísticas descriptivas de los resultados de la muestra y los coeficientes de correlación entre las dimensiones transversales descritas en la muestra. La significancia estadística de estas correlaciones fue establecida con un valor $\mathrm{a}=0,05$.

\section{RESULTADOS}

El ancho transpalatino presentó un valor promedio de 35,72 cm (DS, 1,96), el ancho maxilar presentó un valor promedio de $64,58 \mathrm{~cm}$ (DS, 2,8). El ancho

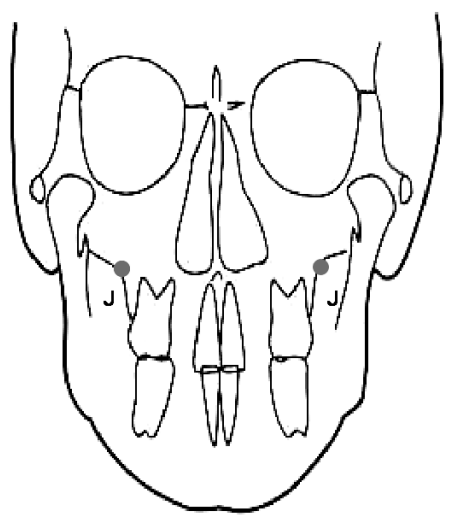

Fig. 1. A. Ancho maxilar.

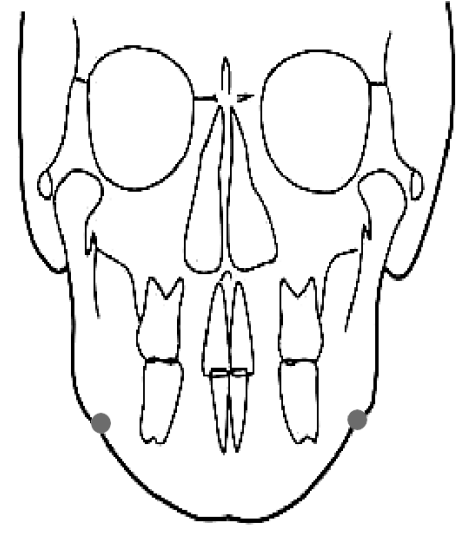

B. Ancho mandibular.

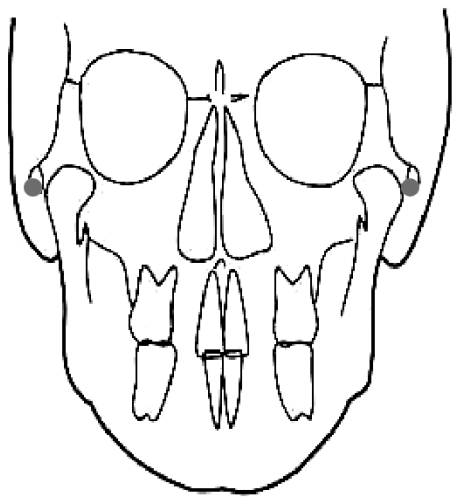

C. Ancho facial. 
mandibular presentó un valor promedio de 79,72 (DS, 3,5), El ancho facial presentó un valor promedio de 121,82 (DS, 4,27).

Una correlación mediana se encontró entre el ancho transpalatino y el ancho maxilar $(r=0,55)$ y entre el ancho maxilar y el ancho facial $(\mathrm{r}=0,54)$, estas relaciones resultaron estadísticamente significativas $(a=0,05)$. Las restantes relaciones resultaron tener una baja correlación, siendo mínima entre el ancho transpalatino y el ancho facial $(r=0,01)$.
Los valores de los coeficientes de correlación se resumen en la Tabla I.

Al analizar la muestra según sexo, se observan valores promedios mayores en hombres que en mujeres para los anchos facial, maxilar y mandibular. No obstante, el ancho transpalatino resultó levemente mayor en mujeres. Ninguna de las diferencias encontradas resultó estadísticamente significativa. Los valores promedio y desviaciones estándares se presentan en la Tabla II.

Tabla I. Coeficientes de correlación r, entre las medidas de los anchos transpalatino, facial, maxilar y mandibular, en 45 pacientes (22 de sexo femenino y 23 de sexo masculino) con edades de entre 7 y 10 años, de la Clínica del Niño del Centro de Clínicas Odontológicas de la Universidad de Talca, Chile, diagnosticados previamente como individuos mesofaciales.

\begin{tabular}{lccc} 
& Ancho transpalatino & Ancho facial & Ancho maxilar \\
Ancho facial & 0.010224 & & \\
Ancho maxilar & 0.558617 & 0.545854 & 0.107327 \\
Ancho mandibular & 0.159422 & 0.23702 & \\
\hline
\end{tabular}

Tabla II. Valores promedio y desviación estándar ( $\mathrm{mm}$ ) de los anchos transpalatino, maxilar, mandibular y facial en 45 pacientes (22 de sexo femenino y 23 de sexo masculino) con edades de entre 7 y 10 años, de la Clínica del Niño del Centro de Clínicas Odontológicas de la Universidad de Talca, Chile, diagnosticados previamente como individuos mesofaciales.

\begin{tabular}{ccccc}
\hline & Ancho transpalatino & Ancho maxilar & Ancho mandibular & Ancho facial \\
Hombres & $35,5 \pm 2,38$ & $65.1 \pm 2,89$ & $80.5 \pm 3,66$ & $122.9 \pm 4,02$ \\
Mujeres & $35,9 \pm 1,45$ & $64.1 \pm 2,70$ & $79.0 \pm 3,27$ & $120.7 \pm 4,32$ \\
\hline
\end{tabular}

\section{DISCUSIÓN}

En el presente estudio fueron analizados los valores de los anchos transpalatino, maxilar, mandibular y facial y las correlaciones existentes entre ellos.

Se encontró que la mayoría de los niños presentaban el ancho transpalatino entre 35,6 y $39 \mathrm{~mm}$, lo que representaba el 52,1\%; el 43,8\% presentaba el ancho transpalatino entre 32 y $35,5 \mathrm{~mm}$ y sólo el $4,2 \%$ presentaba el ancho transpalatino entre 39,1 y $42,5 \mathrm{~mm}$.

Un estudio similar fue realizado por Spillane \& McNamara, quienes analizaron el ancho transpalatino en niños caucásicos a los 9 años de edad, tomando como puntos de referencia los mismos parámetros utilizados en el presente estudio. Los rangos de valores que obtuvieron fueron: < $31 \mathrm{~mm}$; entre 31 y $35 \mathrm{~mm}$ y $>$ a 35 . En la presente investigación no se encontró ningún caso donde el ancho transpalatino fuese menor a $31 \mathrm{~mm}$ y sólo el 43,8\% (21 casos) se encontraron en el rango de 31 a $35 \mathrm{~mm}$.
No se observaron diferencias significativas atribuibles al sexo en los valores obtenidos lo que probablemente se debe al grupo etáreo estudiado, Moyers manifiesta que los incrementos de arco se presentan fundamentalmente durante la erupción activa de los dientes en el cambio de la dentición temporal por la permanente; así mismo, Athanasiou et al. (1992) manifestaron que el ancho intermolar maxilar entre $\operatorname{los} 9$ y 12 años no presenta incremento alguno. Por otra parte, Sinclair \& Little (1983) confirmaron que el aumento del ancho intermolar posterior a los 12 años, es estadísticamente diferente entre hombres y mujeres. De Koch (1972) apoya esta afirmación manifestando que posterior a los 12 años, el crecimiento de ancho de arco solamente se presenta en individuos de sexo masculino. Algunos estudios por tanto, como el de Scheideman et al. (1980) que estudiaron un grupo de adultos caucásicos, concluyeron que existen diferencias entre ambos sexos y que éstas son significativas, al igual que lo observado por Águila (2000) y Moyers quienes obtuvieron valores superiores para el ancho transpalatino en el sexo masculino. 
Los valores encontrados en este estudio difieren de los obtenidos por Ricketts en una población de niños caucásicos de la misma edad. Ricketts presentó como norma clínica para el ancho maxilar el valor de $61,9 \mathrm{~mm}+0,6$ $\mathrm{mm}$. Snodell et al., estudiaron los cambios longitudinales en sujetos caucásicos, encontrando que el ancho maxilar en niñas fue de 58,1 mm y en niños de $60,0 \mathrm{~mm}+0,5 \mathrm{~mm}$, ambos resultados muestran valores inferiores a los obtenidos en el presente estudio. Cabe señalar que en la presente investigación, el valor promedio fue mayor en el sexo masculino $(65,01 \mathrm{~mm})$ que en el femenino $(64,1 \mathrm{~mm})$.

En el estudio realizado por Ricketts la media para el ancho facial fue de $115,7 \mathrm{~mm}+2,4 \mathrm{~mm}$, mientras que en el estudio realizado por Snodell et al. los valores promedio fueron de $114,17 \mathrm{~mm} \pm 1,4 \mathrm{~mm}$ para el sexo femenino y
$117,1 \mathrm{~mm} \pm 1,2 \mathrm{~mm}$ en el sexo masculino, valores que también resultan inferiores a los del presente estudio. Estas diferencias pueden atribuirse a que en nuestra muestra se incluyeron sólo individuos mesofaciales.

Finalmente, este estudio establece que en la muestra de 45 pacientes mesofaciales de la Región del Maule del grupo etáreo seleccionado, la medida del ancho transpalatino mostraba una mediana correlación con el ancho maxilar, no así con el ancho facial, por lo que individuos clínicamente braquifaciales o dolicofaciales pueden pertenecer a una clasificación morfofacial distinta a nivel esqueletal, por tanto los valores de los anchos transpalatino y maxilar medido, en osamentas no serían buenos indicadores para estimar el tipo facial con fines forenses, en este grupo etáreo.

RAMOS, P. N. A.; SUAZO, G. I. C.; MARTINEZ, M. M. L. \& REYES, L. Transverse face relations in chilean children from Maule Region. Int. J. Morphol., 25(4):703-707, 2007.

SUMMARY: The transverse face relations present variations in the different ethnic groups, their knowledge is excellent in the field of the plastic and maxilofacial surgery, the anthropology and the forensic medicine. In the present study the face cross-sectional relations of 45 mesofacial children of the maule region by means of a frontal cephalometric analysis and the preparation of models of paladar are analyzed. The dimensions were analyzed: transpalate wide, maxilar wide, face wide and mandibular wideThe found dimensions were superior to the described thing in Literature. The transpalate wide showed a medium correlation with the maxilar wide, but not thus with the maxilar wide. Not sexual dimorphism was observed in the sample.

KEY WORDS: Relations face; Face; Physical Antrophology; Forence Medicine.

\section{REFERENCIAS BIBLIOGRÁFICAS}

Águila, J. Manual de Cefalometría. Barcelona, Actualidades Médico Odontológicas, 1998.

Águila, J. Tratado de Ortodoncia. 33 . ed. Barcelona, Actualidades Médico Odontológicas, 2000.

Athanasiou, A. E.; Droschl, H. \& Bosch, C. Data and patterns of transverse dentofacial structure of 6 to 15 year old children: a posteroanterior cephalometric study. Am. J. Orthod. Dentofacial. Orthop., 101(5):465-71, 1992.

Björk, A. The principle of the Andresen method of orthodontic treatment a discussion based on cephalometric x-ray analysis of treated cases. Am. J. Orthod. Dentofacial. Orthop., 37(7):437-58, 1951.

Burris, B. G. \& Harris, E. F. Maxillary arch size and shape in American blacks and whites. Angle Orthod., 70(4):297-302, 2000.

Chaconas, S. Ortodoncia. México El Manual Moderno, 1982.
De Koch, W. H. Dental arch depth and width studied longitudinally from 12 years of age to adulthood. Am. J. Orthod. Dentofacial. Orthop., 62(1):56-66, 1972.

Del Sol, M. Indice cefálico en un grupo de individuos mapuches de la IX Región de Chile. Int. J.Morphol., 23(3):241-6, 2005.

Fonseca, R. J. \& Klein, W. D. A cephalometric evaluation of American Negro women. Am. J. Orthod. Dentofacial. Orthop., 73(2):152-60, 1978.

García, H. F. \& Lips, M. W. Contribución al estudio del índice cefálico en chilenos. An. Anat. Normal, 4:120-3, $1986 a$.

García, H. F. \& Lips, M. W. Variaciones del índice cefálico en chilenos según ascendencia. An. Anat. Normal, 4:1179, $1986 b$.

Ghafari, J.; Cater, P. E. \& Shofer F. S. Effect of film object 
distance on posteroanterior cephalometric measurements: suggestions for standardizes cephalometric methods. Am. J. Orthod. Dentofacial Orthop., 108(1):30-7, 1995.

Gravilovic, Z. L'indice cephalique chez le yougoslaves. Comp. Rend. Assoc. Anat., 138:552-8, 1967.

Gregoret, J. Ortodoncia y cirugía ortognática, Diagnóstico y planificación de tratamiento. Barcelona, Publicaciones Médicas, 1997.

Grummons, D. \& Kappeyne van de Coppello, M. A frontal asymmetry analysis. J. Clin. Orthod. 21(7):448-65, 1987.

Gundermann, H.; Vergara, J. \& Foerster, R. Contar a los indígenas en Chile. Autoadscripción étnica en la experiencia censal de 1992 y 2002. Estudios Atacameños, 30:91$115,2005$.

Huertas, D. \& Ghafari, J. New posteroanterior cephalometric norms: a comparison with craniofacial measures of children treated with palatal expansion. Angle. Orthod., 71(4):285-92, 2001.

Jacobson, B. N. \& Rosestein, S. W. Cephalometry: an important orthodontic tool. Inf. Orthod. Kieferorthop., 2(3):36-52, 1970.

Kowalski, C. J.; Nasjleti, C. E. \& Walker, G.F. Differential diagnosis of adult male black and white populations. Angle Orthod., 44(4):346-50, 1974.

Moyers, R. E. Manual de Ortodoncia. 4a ${ }^{\mathrm{a}}$. ed. Buenos Aires, Editorial Médica Panamericana, 1992.

Quevedo, L. \& Jeldes, G.; Análisis cefalométricos y estéticos más utilizados en planificación de tratamiento para cirugía ortognática. $1^{\text {a }}$. ed. Universidad de Chile, Facultad de Odontología, Santiago-Chile, 2004.

Ricketts, R. M. Perspectives in the clinical application of cephalometrics. The first fifty years. Angle Orthod., 51(2):115-50, 1981.

Scheideman, G. B.; Bell, W. H.; Legan, H. L.; Finn, R. A. \& Reisch, J. S. Am. J. Orthod. Dentofacial. Orthop., 78(4):404-20, 1980.

Sinclair, P. M. \& Little, R. M. Maturation of untreated normal occlusions. Am. J. Orthod. Dentofacial. Orthop., 83(2):114-23, 1983.
Snodell, S. F.; Nanda, R. S. \& Currier, G. F. A longitudinal cephalometric study of transverse and vertical craniofacial growth. Am. J. Orthod. Dentofacial. Orthop., 104(5):471-83, 1993.

Spillane, L. M.; McNamara, J. A. Arch width development relative to initial transpalatal width. J. Dent. Res. Abst., 68(1538):374, 1989.

Vellini Ferreira, F. Ortodoncia - Diagnóstico y planificación Clínica. $1^{\mathrm{a}}$. ed. São Paulo, Artes Médicas Latinoamericanas, 2002.

Dirección para correspondencia:

Prof. Dr. Iván Suazo Galdames

Unidad de Anatomía Normal

Universidad de Talca

Avenida Lircay s/n oficina $N^{\circ} 104$

Talca - CHILE

Fono 56-71-201682

Email: isuazo@utalca.cl niramos@utalca.cl

Recibido : 08-06-2007

Aceptado: 12-08-2007 
